\title{
A New Novel Fidelity Digital Watermarking Adaptively Pixel Based on Medial Pyramid of Embedding Error in Spatial Domain and Robust
}

\author{
Mehemed Bashir Aliwa, Tarek El-Ahmady El-Tobely, Mahmoud M. Fahmy, Mohamed EL Said Nasr, \\ and Mohamed Hashem Abd El-Aziz
}

\begin{abstract}
Digital watermarking refers to techniques that are used to protect digital data by imperceptibly embedding watermark into the original data in such a way that always remains present. In particular, digital watermarking techniques in frequency domain have been widely recognized to be more prevalent than others, but in recent years the techniques in spatial domain they are becoming generally abandoned. One of the problems in digital watermarking is that the three requirements of robustness capacity and imperceptibility, that are must be satisfied but they almost conflict with each other, accordingly there are trade-off between fidelity and robustness. In this paper, we proposed a new novel fidelity and robust watermark embedding method that satisfies the requirements and statement problem, called adaptively pixel adjustment process based on medial pyramid of embedding error, applying in the falling-off-boundary in corners board of the cover image set of the Most Significant Bit ' 6 ' blind in spatial domain. In addition, the paper provides a theoretical analysis and modified algorithms of previous works. Theoretically, the proposed technique proves the effectiveness of the technique in the average of worst case and minimizing the number of embedding error to the half. Experimental results of the proposed technique was applied on the different benchmark of six gray scale images and two quantum of watermark bit embedded are compared with previous works and was found better. Moreover in all different benchmark of six test images the watermarks were extracted from watermark degrading, removal and geometric transformations attacks to an acceptable degree.
\end{abstract}

Index Terms-Fidelity, digital watermarking, imperceptible, spatial domain, LSB \& MSB, benchmark.

\section{INTRODUCTION}

Digital watermarking is a technique which allows an individual to add hidden copyright or other messages to digital audio, video, or image signals [1], [2]. The important watermarking characteristics are exhibit. Imperceptibility: means that the perceived quality of the host image should not be distorted by the presence of the watermark [3]. Fidelity: refers to the term imperceptible as it is referred in the literature of watermarks [4]. Capacity: knowing how much information can reliably be hidden in the signal [2] and refers

Manuscript received November 21, 2012; revised January 24, 2013.

Mehemed Bashir Aliwa, Tarek El-Ahmady El-Tobely, Mahmoud M. Fahmy, and Mohamed EL Said Nasr are with the Faculty of Engineering-TANTA University, in Egypt (e-mail: aliwa97@ hotmail.com, tarekeltobely@yahoo.com, mfn-288@ hotmail.com, menasr2001@hotmail.com).

Mohamed Hashem Abd El-Aziz is with the Faculty of Computers and Information Sciences-Ain Shams University, in Egypt (e-mail: mhashem100@yahoo.com). to the bit size of a payload that a watermark access unit can carry[5] or how many marks can be added simultaneously[6], [7]. Data payload: refers to the amount of information stored in the watermark [4]. Robustness: The ability of the watermark to survive normal processing of content [8]. Data secrecy: For more protection to the watermark bits a secret-Key has been used to permute the watermark bits before embedding it to achieve cryptographic security [9]. Redundancy: To ensure robustness, the watermark information is embedded in multiple places on the cover data file [2], [8].

The paper is organized as follows. Section II describes the statement problem. In Section III describes the proposed modified previous works. In Section IV describes the proposed watermarking technique. In Section V describes the experimental results. Finally conclusion.

\section{Statement Problem In Spatial Domain}

The digital watermarking technology is a way to apply digital information hiding techniques to prevent attacks to detect hidden information. In particular, digital watermarking techniques in frequency domain have been widely recognized to be more prevalent than others [3] but in recent years the techniques in spatial domain they are becoming generally abandoned [10]. The problem in digital watermarking is that there are three requirements of imperceptibility, capacity, and robustness which must be satisfied but they almost always conflict with each other, in the same case there are trade-off between fidelity and robustness. Accordingly, the proposed solution is to embed a watermark image within the pixels of the cover image in spatial domain, but still there is another problem, (i): when an image is being embedded, it shouldn't cause any visual change to the cover image, whereas almost techniques using a Least-significant-bit(LSB) in spatial domain to hide a watermark image[6], [9], [11]-[14] or massage within a low embedding errors, where the authors are avoiding to use the Most-Significant-Bit (MSB). While the statement problem there are a trade-off between the embedding error in the LSB and MSB. Furthermore the embedding process in the LSB do not introduce any perceptible into the cover image, as well as the embedding errors in the LSB growth up from $\left(1_{\text {Min }}\right.$ to $\left.8_{\text {Max }}\right)$, while in the MSB growth up from ( $16_{\text {Min }}$ to $\left.128_{\text {Max }}\right)$, with introducing higher perceptible into the cover image. On the other hand the authors investigated into the use of the LSB substitution technique in digital watermarking [14], the LSB embedded 
watermark bits can easily be removed using techniques, that do not affect the image visually to the point of being noticeable and if the watermark is hidden in the LSB, all the individual has to do is flip one LSB, thus the information cannot be recovered, that why in recent years the techniques in spatial domain they are becoming generally abandoned. (ii): Another problem appears with this since the image is limited by its dimensions, the number of bits that are usable for embedding is also limited and the watermark image should be chosen in such that it could fit in the cover image. From these problems we aim at introducing to development an enhanced approach for digital watermarking for hiding information that is satisfies these requirements and problems at the same time in an acceptable manner.

\section{Proposed ModifiEd PREVIOUS WORKS}

To analysis study the performance and comparisons between the state-of-the-art algorithms will be modified the algorithms of pixel adjustment process (PAP) are based in the LSB techniques are proposed [9], [11], [13], [14], after that will be applying the modification algorithms of PAP by the our embedding algorithm of the FOBCB set-of-the-MSB 6 [15], the Max-of embedding errors in the $\mathrm{MSB}_{6}$ by directly replacement of embedding watermark bits $=2^{n-1}=32$. Moreover there is a trade-off between the embedding errors in the LSB and MSB, where the embedding errors are growth up as $=2^{n-1}$. Let's have the binary watermark image $W L_{(i i, j j)}$ a size of $W L=[T, U]$, and the cover image $F=\left\{\right.$ pixel $_{1}$, pixel $_{2}, \ldots$, $\left.\operatorname{pixel}_{(M \times N)}\right\}=P_{(i, j)}$, after extracted the pixels from cover image, will be converted the cover image pixels $P_{(i, j)}$ in to the binary numbers ( 8 bits per pixel), then set of the $\mathrm{MSB}_{6}$ in each pixel of cover image $P_{(i, j)}$ accounted from right to the left hand, the following proposed modified algorithms in $\mathrm{MSB}_{\mathrm{n}}$ and our analysis computed under all possibility gray-scale-values:

\section{A. PAP-Algorithm-1}

We modified the scheme of Wang-Lin-Lin [11] using a local pixel adjustment process (LPAP) the proposed algorithm used $\mathrm{LSB}_{4}$ for embedding data bits, thus will be modified the algorithm of LPAP on the $\mathrm{MSB}_{6}$. However the embedding error in $\mathrm{MSB}_{6}$ equal 32 was trade-off with the embedding error in $\mathrm{LSB}_{4}$ equal 8 . Let $P_{(i, j)}$ a pixel of cover image and $p_{(i, j)}$ watermarked image obtained by applying FOBCB set-of-MSB ${ }_{6}$ scheme[15], respectively, and $\delta$ be the value of the $\left(\mathrm{LSB}_{1,2,3,4} \& \mathrm{MSB}_{5}\right)$ as well as from $\left\{\right.$ bit $_{1}$ to $\left.\mathrm{bit}_{5}\right\}$ in $p^{\prime}{ }_{(i, j)}$. If $P_{(i, j)} \neq p^{\prime}{ }_{(i, j)}$, then either (i): $p^{\prime}{ }_{(i, j)}=P_{(i, j)}-2^{n-1}$ or (ii): $p^{{ }_{(i, j)}}=P_{(i, j)}+2^{n-1}$. Case 1: when $p^{{ }_{(i, j)}}=P_{(i, j)}-2^{n-1}$. If $\delta \geq 2^{n-2}$, then the value $\left(2^{n-1}-\delta-1\right)$ is added to $p^{\prime}{ }_{(i, j)}$. If $\delta<2^{n-2}$ and if the seven bit of $p^{\prime}{ }_{(i, j)}$ is zero, then the seven bit of $p^{{ }_{(i, j)}}$ is changed to one, and the value $\delta$ is subtracted from $p^{\prime}{ }_{(i, j)}$. Do nothing otherwise. Case 2: when $p^{\prime}{ }_{(i, j)}=P_{(i, j)}+2^{n-1}$. If $\delta<2^{n-2}$, then the value $\delta$ is subtracted from $p^{\prime}{ }_{(i, j)}$. If $\delta \geq 2^{n-2}$ and if the seven bit of $p^{\prime}(i, j)$ is one, then the seven bit of $p^{\prime}{ }_{(i, j)}$ is changed to zero, and the value $\left(2^{n-1}-\delta-1\right)$ is added to $p^{\prime}{ }_{(i, j)}$. Do nothing otherwise. Theoretical analysis: Notice that from the PAP of the Wang-Lin-Lin scheme, we know that only the first three bits (bits 1-3) and the five bit $\left(\mathrm{MSB}_{5}\right)$ are modified. It is obvious that the algorithm is not optimal if $P_{(i, j)}=8,15,24$, 25,250 ; when the embedded watermark bit equal zero, whereas the embedding error go to level of Max-error in the $\mathrm{LSB}_{4}=2^{n-1}=8$, However, it can be seen that the embedding error go to the Min-error if $P_{(i, j)}=11,12,28,75,236$; when the embedded watermark bit equal zero, the embedding error go to the Min-level error in $\mathrm{LSB}_{4}=\left(2^{n-2}+1\right)=5$. Also the same problem of modified (PAP-algorithm-1) set-of the $\mathrm{MSB}_{6}$. It is obvious that the modification is not optimal where the embedding error are confined between the Max-level of the embedding error in $\operatorname{MSB}_{6}=\left(2^{n-1}\right)=32$ and in the Min-level of the embedding error in $\operatorname{MSB}_{6}=\left(2^{n-2}+1\right)=17$. Thus the observation of the analysis result in the both algorithms the embedding error are growth up one by one start from $\left(2^{n-2}+1\right)$ to the $\left(2^{n-1}\right)_{\text {Max }}$. Theoretically, can be calculated the average of embedding errors between the Max and Min number in both algorithms, then the number ' $i$ ' of embed errors, can be derived by:

$$
\begin{aligned}
& \sum_{i=1}^{2^{n-2}} i=1+2+\ldots+2^{n-2}=\frac{2^{n-2}\left(2^{n-2}+1\right)}{2}=2^{n-3}\left(2^{n-2}+1\right) \\
& \sum_{i=1}^{2^{n-1}} i=1+2+\ldots+2^{n-1}=\frac{2^{n-1}\left(2^{n-1}+1\right)}{2}=2^{n-2}\left(2^{n-1}+1\right)
\end{aligned}
$$

Subtracting Eqs.(1) and (2), then we get the summation of the embedding errors ' $i$ ':

$$
2^{2 n-3}+2^{n-2}-2^{2 n-5}-2^{n-3}=2^{n-2}\left(2^{n-1}-2^{n-3}+1\right)-2^{n-3}
$$

From Eqs.(3) the average of embedding errors in both algorithms can be derived by Eqs.(4):

$$
\begin{aligned}
\text { The average of embedding error } & =\frac{2^{n-2}\left(2^{n-1}-2^{n-3}+1\right)-2^{n-3}}{2^{n-2}} \\
& =\frac{2\left(2^{n-1}-2^{n-3}+1\right)-1}{2}
\end{aligned}
$$

Then can be computed the mean square error MSE given by formula Eq.(5). Theoretically, in the average of worst mean square error Averg.WMSE in both algorithms are derived by Eqs. $(4,5)$ as:

$$
\begin{gathered}
M S E=\frac{1}{M \times N} \sum_{i=1}^{M} \sum_{j=1}^{N}\left(F_{(i, j)}-f_{(i, j)}\right)^{2} \\
\text { Averg.WMSE }{ }^{*}=\frac{1}{M \times N} \sum_{i=1}^{M} \sum_{j=1}^{N}\left[\frac{2\left(2^{n-1}-2^{n-3}+1\right)-1}{2}\right]^{2} \\
\text { Averg.WMSE }{ }^{*}=\left[\frac{2\left(2^{n-1}-2^{n-3}+1\right)-1}{2}\right]^{2}
\end{gathered}
$$

From the above Eq. (6). Theoretically, can be derived by Eq.(5) as well as the Max.WMSE ${ }^{*}=\left(2^{n-1}\right)^{2}$ and the Min. WMSE ${ }^{*}=\left(2^{n-2}+1\right)^{2}$ are obtained after applying modified algorithm, thus the PSNR ${ }_{\text {worst }}$ are obtained as:

$$
P S N R_{\text {worst }}=10 \times \log _{10} \frac{255^{2}}{W M S E^{*}} d B
$$

\section{B. PAP-Algorithm-2}

We modified the Chi-Kwong-L. M. Cheng [13] scheme using optimal pixel adjustment process (OPAP) the proposed embedding algorithm in the $\mathrm{k}$, means capacity of embedding 
data bit in $k-\mathrm{LSB}_{n}$ of the cover image, where the Max-embedding errors growth up respectively from $\{1,3$, 7 and $\left.15_{\text {Max }}\right\}$ depending in the value of $\mathrm{k}$, then will be applying the OPAP algorithm in the $\mathrm{MSB}_{6}$ without using k of capacity. Thus the embedding errors in $\mathrm{MSB}_{6}=32$ are greater than with compared by using a capacity of $\mathrm{k}$ bits in $\left(\mathrm{LSB}_{1,2,3,4}\right)$ are equal 15. Let is $P_{(i, j)}, P_{(i, j)}^{\prime}$ and $P_{(i, j)}^{\prime \prime}$ be the corresponding pixel values of a pixel in the cover image, the embedding image $P_{(i, j)}^{\prime}$ obtained by applying the embedding algorithm FOBCB set-of-MSB ${ }_{6}$ scheme[15] and the refined embedding image obtained after the modified PAP-algorihm-2 $P_{\left({ }_{(i, j)}\right)}$. Let absolute $\delta_{(i, j)}=\left|P_{(i, j)}^{\prime}-P_{(i, j)}\right|$ be the embedding error between $P_{(i, j)}$ and $P_{(i, j)}^{\prime}$, therefore, $-2^{n}<\delta_{(i, j)}<2^{n}$, the value of $\delta_{(i, j)}$ can be further segmented into three intervals, such that: Interval-1: $2^{n-1}<\delta_{(i, j)}<2^{n}$. Interval-2: $-2^{n-1} \leq \delta_{(i, j)} \leq 2^{n-1}$. Interval-3: $-2^{n}<\delta_{(i, j)}<-2^{n-1}$. The PAP-algorithm-2 based on the three intervals, which modifies $P_{(i, j)}^{\prime}$ to form the embedding pixel $P^{\prime \prime}{ }_{(i, j)}$, described as: Case 1: $\left(2^{n-1}<\delta_{(i, j)}<2^{n}\right)$ : If $P_{(i, j)}^{\prime} \geq 2^{n}$, then $P^{\prime \prime}{ }_{(i, j)}=P_{(i, j)}^{\prime}-2^{n}$; otherwise $P_{(i, j)}^{\prime \prime}=P_{(i, j)}^{\prime} ;$ Case $2:\left(-2^{n-1} \leq \delta_{(i, j)} \leq 2^{n-1}\right)$ : $P^{\prime \prime}{ }_{(i, j)}=P_{(i, j)}^{\prime}$; Case 3: $\left(-2^{n}<\delta_{(i, j)}<-2^{n-1}\right)$ : If $P_{(i, j)}^{\prime}<256-2^{n}$, then $P^{\prime \prime}{ }_{(i, j)}=P_{(i, j)}^{\prime}+2^{n}$; otherwise $P^{\prime \prime}{ }_{(i, j)}=P_{(i, j)}^{\prime}$. Where the $P_{(i, j)}^{\prime \prime}$ are obtained by FOBCB set-of-MSB 6 with applying PAP-algorithm-2 and the embedding error between $P_{(i, j)}$ and $P^{\prime \prime}{ }_{(i, j)}$ computed by $\delta_{(i, j)}^{\prime}=\left|P_{(i, j)}^{\prime \prime}-P_{(i, j)}\right|$. Theoretical analysis: from the Chi-K.scheme(OPAP)[13] the algorithm minimized the embedding error from $\left(2^{k}-1\right)$ to $2^{k-1}$. Theoretically, can be calculated the number of embedding errors ' $i$ ' are start from ' 1 ' to $=2^{k-1}$, can be derived by:

$$
\sum_{i=1}^{2^{k-1}} i=1+2+\ldots+2^{k-1}=\frac{2^{k-1}\left(2^{k-1}+1\right)}{2}=2^{k-2}\left(2^{k-1}+1\right)
$$

From Eq.(9) the average of embedding errors with k-LSB are derived by Eq.(10):

$$
\text { The average of embedding error }=\frac{2^{k-2}\left(2^{k-1}+1\right)}{2^{k-1}}=\frac{2^{k-1}+1}{2}
$$

From Eqs. $(5,10)$ the averg.WMSE can be derived by:

$$
\text { Averg. } \mathrm{WMSE}^{*}=\frac{1}{M \times N} \sum_{i=1}^{M} \sum_{j=1}^{N}\left[\frac{\left(2^{k-1}+1\right)}{2}\right]^{2}=\left[\frac{\left(2^{k-1}+1\right)}{2}\right]^{2}
$$

For example if $P_{(i, j)}=255 \& k=2$, when the embeds watermark bit equal zero, the embedding error go to the level of Max-error in $\operatorname{LSB}_{1,2}=\left(2^{k}-1\right)=3$. Since that our observations, where the embedding error goes to half $(1 / 2)$ of the Max-embedding errors added to half $(1 / 2)$, then the Max-embedding errors of proposed [13] scheme are $=\frac{2^{k}-1+1}{2}=2^{k-1}$ and the averg. WMSE are obtained by

Eq.(3.11). Furthermore by using the same algorithm in [13] by modified the algorithm called PAP-algorithm-3 set-of-MSB $\mathrm{B}_{\mathrm{n}}$ where $n=k$, in this modification the embed watermark bit adjust only embeds one bit in each pixel of the cover image, the Max-of-embedding errors $=2^{n-1}=32$, by applying the PAP-algorithm-3, the embedding errors are always great constant to the Max-level in all cases $=2^{n-1}$. Theoretically the WMSE are constant $\mathrm{WMSE}^{*}=\left(2^{n-1}\right)^{2}$, after applying the PAP-algorithm-3, from $\operatorname{Eqs}(5,7)$ calculated the PSNR $_{\text {worst. }}$

\section{PAP-Algorithm-3}

We modified the algorithm of Aiad and Abdul [9] using local pixel adjustment process (LPAP) the proposed algorithm used $\mathrm{LSB}_{3}$ to embedded message bit with modified $\mathrm{LSB}_{1,2}$ according to the embedding data bit in $\mathrm{LSB}_{3}$. So that will be modified the algorithm of LPAP in to $\mathrm{MSB}_{6}$ by applying on the embedding algorithm FOBCB set-of-MSB ${ }_{6}[15]$, Let's have the cover image $P_{(i, j)}$. Suppose that $\mathrm{MSB}_{6}$ of the cover image is $\mathrm{MSB}_{6}=\left\{\mathrm{MSB}_{1}\right.$, $\left.\mathrm{MSB}_{2}, \mathrm{MSB}_{3}, \ldots, \mathrm{MSB}_{(N \times M)}\right\}$, where $\mathrm{MSB}_{6}=\{0,1\}$. The embedding process of the watermark bit $(\mathrm{EMB})$ by applying the embedding algorithm FOBCB set-of-MSB 6 of the cover image to obtain the new embedding image $=\left\{\right.$ newpixel $_{(1,1)}$, newpixel $_{(2,1)}, \ldots$, newpixel $\left.l_{(N, M)}\right\}$. The following embedding algorithm of LPAP set-of-MSB 6 : Step 1: Extract $\mathrm{LSB}_{1}$ set of the cover image, $\mathrm{LSB}_{1}=\left\{\mathrm{LSB}-1_{1}, \mathrm{LSB}-1_{2}, \ldots, \mathrm{LSB}-1_{L}\right\}$. Step2: Extract $\mathrm{LSB}_{2}$ set of the cover image, $\mathrm{LSB}_{2}=\left\{\mathrm{LSB}-2_{1}, \mathrm{LSB}-2_{2}, . ., \mathrm{LSB}-2_{L}\right\}$. Step3: Extract $\mathrm{LSB}_{3}$ set of the cov-image, $\mathrm{LSB}_{3}=\left\{\mathrm{LSB}-3_{1}, \mathrm{LSB}-3_{2}, \ldots, \mathrm{LSB}-3_{L}\right\}$. Step4: Extract $\mathrm{LSB}_{4}$ set of the cover image, $\mathrm{LSB}_{4}=\left\{\mathrm{LSB}-4_{1}\right.$, LSB- $\left.4_{2}, \ldots, \mathrm{LSB}-4_{L}\right\}$. Step5: Extract $\mathrm{MSB}_{5}$ set of the cover image, MSB $_{5}=\left\{\mathrm{MSB}-5_{1}, \quad \mathrm{MSB}-5_{2}, \ldots, \mathrm{MSB}-5_{L}\right\} . \quad$ Step6: Extract $\mathrm{MSB}_{6}$ set of the cover image, $\mathrm{MSB}_{6}=\left\{\mathrm{MSB}-6_{1}\right.$, MSB- $6_{2}, \ldots, \mathrm{MSB}_{2} 6_{L}$. Step7: Set watermark $\mathrm{WL}=\left\{\mathrm{EMB}_{1}, \mathrm{EMB}_{2}, \ldots . ., \mathrm{EMB}_{(T \times U)}\right\}$.

Step8: For $i i=1$ to $T$

For $j j=1$ to $U$

if $\mathrm{MSB}_{6(i i, j j)}==\mathrm{EMB}_{(i i, j j)}$, then do nothing

$\operatorname{MSB}_{6(i i, j j)}=\mathrm{EMB}_{(i i, j j)}$;

else if $\operatorname{MSB}_{6(i i, j j)}==0$ and $\mathrm{EMB}_{(i i, j j)}==1$, then

$$
\begin{aligned}
& \mathrm{LSB}_{1(i i, j j)}=0 ; \mathrm{LSB}_{2(i i, j j)}=0 ; \mathrm{LSB}_{3(i i, j j)}=0 \text {; } \\
& \mathrm{LSB}_{4(i i, j j)}=0 ; \mathrm{MSB}_{5(i i, j j)}=0 ; \mathrm{MSB}_{6(i i, j j)}=\mathrm{EMB}_{(i i, j j)} \text {; } \\
& \text { else if } \operatorname{MSB}_{6(i i, j j)}==1 \text { and } \operatorname{EMB}_{(i i, j j)}==0 \text {, then } \\
& \mathrm{LSB}_{1(i i, j j)}=1 ; \mathrm{LSB}_{2(i i, j j)}=1 ; \mathrm{LSB}_{3(i i, j j)}=1 \text {; } \\
& \operatorname{LSB}_{4(i i, j j)}=1 ; \mathrm{MSB}_{5(i i, j j)}=1 ; \operatorname{MSB}_{6(i i, j j)}=\mathrm{EMB}_{(i i, j j)} \text {; } \\
& \} ;\} ;\} ;\} \text { \}. }
\end{aligned}
$$

Theoretical analysis: Notice that from the algorithm LPAP of the Aiad and Abdul scheme; we know that only the first two bits (bits1-2) are modified. It is obvious that the modification are minimized the embedding errors, if $P_{(i, j)}=7,15,23,31,47$; when the embedded watermark bit equal zero, the embedding error growth up to the Max-error in $\mathrm{LSB}_{3}=2^{n-1}=4$. It is obvious that the modification is not decrease the embedding error where are restricted between a ' $1_{\text {Min }}$ ' and ' $4_{\text {Max }}$ '. Moreover the same procedures applied on the modification PAP-algorithm-3 set-of-MSB ${ }_{6}$ we seen that the embedding errors growth up to the high in $P_{(i, j)}=63,191,255$, when the embeds watermark bit equal zero, the embedding error greats to the Max-error in $\mathrm{MSB}_{6}=2^{n-1}=32$. Our observation from the analysis result of both algorithms the embedding error are growth up one by one start from ' $1_{\text {Min }}$ ' to the Max-embedding error $=2^{n-1}$ and then go down ' 1 ' and then growth up so on. Theoretically, can be calculated the average of embedding errors ' $i$ ' in both algorithms as:

$$
\sum_{i=1}^{2^{n-1}} i=1+2+\ldots+2^{n-1}
$$




$$
\sum_{i=1}^{2^{n-1}} i=2^{n-2}\left(2^{n-1}+1\right)
$$

From Eq.(13) the average of embedding errors in both algorithms can be derived as:

$$
\text { The average of embedding errors }=\frac{\left(2^{n-1}+1\right)}{2}
$$

Theoretically, from the Eq. $(5,14)$ the averg.WMSE in both algorithms derived by:

$$
\text { Averg. } \mathrm{WMSE}^{*}=\frac{1}{M \times N} \sum_{i=1}^{M} \sum_{j=1}^{N}\left[\frac{\left(2^{n-1}+1\right)}{2}\right]^{2}=\left[\frac{\left(2^{n-1}+1\right)}{2}\right]^{2}
$$

Then the Max and Min of WMSE as: Max.WMSE ${ }^{*}=\left(2^{n-1}\right)^{2}$ and Min.WMSE ${ }^{*}=1$ after applying both algorithm, from $\operatorname{Eqs}(5,7,8)$ can be computed the $P S N R_{\text {worst }}$.

\section{PRoposed WATERMARKING TeChNIQUE}

In this section, have been propose a new novel technique of an adaptively pixel adjustment process based on medial pyramid of embedding error APAP-MPOEE set of the MSB by applying in falling-off-boundary in corners board of cover image with the random pixel manipulation blind in spatial domain (APAP-MPOEE-FOBCB ${ }_{\mathrm{MSB} 6}$ ) maybe to enhance the image quality of the watermarked image to great fidelity, robust and imperceptibility. The basic concept of the pixel adjustment process of the $\mathrm{LSB}_{n}$ based on the technique proposed in[9], [11], [13], [14], when $1 \leq n \leq 4$. Hence that the ideas are derive from our study analysis of previous works and modified algorithms. Let $P_{(i, j)}, ' P_{(i, j)}$ and " $P_{(i, j)}$ be the corresponding pixel values of the cover image, ' $P_{(i, j)}$ the watermarked image obtained by applying algorithm $\mathrm{FOBCB}_{\mathrm{MSB} 6}[15]$ and " $P_{(i, j)}$ the refined watermarked image obtained after the applying proposed method (APAP-MPOEE) by FOBCB $_{\text {MSB } 6}[15]$ called APAP-MPOEE-FOBCB MSB6 $_{\text {. }}$ Let's $\Omega^{\prime}=\left|P_{(i, j)}-P_{(i, j)}\right|$ be the embedding error between $P_{(i, j)}$ and ' $P_{(i, j)}$ according to the embedding process of the $\mathrm{FOBCB}_{\mathrm{MSB} 6}$, the following steps of proposed method APAP-MPOEE ${ }_{\mathrm{MSBn}}$, where $6 \leq n \leq 8$.

Step1: Extract pixel from the cover image $P_{(i, j)}$ and converted in to the binary bits $\left(\operatorname{LSB}_{(1,2,3,4)} \& \operatorname{MSB}_{(5,6,7,8)}\right)$, then set of the $\mathrm{MSB}_{6}$ in each pixel within the boundary of corners board, as well as when the $\left\{\mathrm{MSB}_{6}\right.$ of cover image pixel = EMB the embedded watermark bit $\left.W_{(i, j)}\right\}$ then do nothing. Otherwise when the $\mathrm{MSB}_{6}$ in cover image pixel not equal the embedded watermark bit $(\mathrm{EMB}), \mathrm{MSB}_{6} \neq \mathrm{EMB}$, thus the pixel value of cover image $P_{(i, j)}$ can be further segmented into intervals, whereas the Max-pixel value of cover image in interval with 8 bit at in the range $0 \leq P_{(i, j)}<256$, theoretically, can be derived the intervals depending on the embedding error $=\left(2^{n-1}\right)$ in each bit as:

$$
\text { The number of intervals in } \mathrm{MSB}_{\mathrm{n}}=\frac{256}{2^{n-1}}=8
$$

From Eqs.(16) the all number of intervals in $\mathrm{MSB}_{\mathrm{n}}=8, \mathrm{n}=6$. Furthermore will be divided the eight intervals depending on the step of the embedding error Step.2: when $\mathrm{MSB}_{6}={ }^{\prime} 0$ ' and the $\mathrm{EMB}={ }^{\prime} 1$ '. Step3: when $\mathrm{MSB}_{6}={ }^{\prime} 1$ ' and the $\mathrm{EMB}={ }^{\prime} 0$ '.
From step2 and step3, theoretically can be further segmented into four intervals in step2 \& step3 as:

The number of intervals in each $\operatorname{step}_{2,3}=\frac{256}{2^{n}}=4$

Hence that the embedding process in the $\mathrm{MSB}_{6}$ of the cover image pixel in the boundary of corners board to form the watermarked pixel " $P_{(i, j)}$ that required eight intervals as Eq.(16), thus each interval will be divided in to two intervals to minimizing the embedding error in to the medial pyramid of embedding error, then will be get sixteen intervals ' 16 ', can be described as: First: will be divided each interval in to two intervals. Second: Added $\left(2^{n-2}\right)$ in each start interval to get the end of a new interval, where are from the interval-1 will be get two as in case.1. Hence that each interval from (1-8) is divided in to the half $(1 / 2)$ in each interval, to obtained a sixteen intervals " 16 " from case.(1-8) in step:( $2 \& 3)$, can be derived in this step2,3 based on four intervals in each step, the proposed APAP-MPOEE scheme, which the algorithm requires a checking between the value of $\mathrm{MSB}_{6}$ and the value of EMB before embedding the watermark bit depending on the nearest of adaptively pixel in the medial pyramid of embedding error to inform the watermarked image " $P_{(i, j)}$ are described in the following of step 2,3:

Step2: In this step when the $\mathrm{MSB}_{6}=0$ and $\mathrm{EMB}=$ '1', then the value pixels of cover image $P_{(i, j)}$ can be further segmented into four intervals as Eqs. $(17,18)$, such that:

Interval $_{1}: 0 \leq P_{(i, j)}<2^{n-1}$. Interval $2: 2^{n} \leq P_{(i, j)}<3 \times 2^{n-1}$

Interval $_{3}: 2^{n+1} \leq P_{(i, j)}<5 \times 2^{n-1}$. Interval $4: 3 \times 2^{n} \leq P_{(i, j)}<7 \times 2^{n-1}$

From intervals (1-4), the proposed APAP-MPOEE scheme required to divide each interval to the half $(1 / 2)$ derived as:

Case 1: $\left(0 \leq P_{(i, j)}<2^{n-1}\right)$, then if $\left(0 \leq P_{(i, j)}<2^{\mathrm{n}-2}\right)$, then " $P_{(i, j)}=2^{n-1}$; else " $P_{(i, j)}=2^{n-1}$; end.

Case 2: $\left(2^{n} \leq P_{(i, j)}<3 \times 2^{n-1}\right)$, then if $\left(2^{n} \leq P_{(i, j)}<5 \times 2^{n-2}\right)$, then " $P_{(i, j)}=2^{n}-1$; else " $P_{(i, j)}=3 \times 2^{n-1}$; end.

Case 3: $\left(2^{n+1} \leq P_{(i, j)}<5 \times 2^{n-1}\right)$, then if $\left(2^{n+1} \leq P_{(i, j)}<9 \times 2^{n-2}\right)$, then " $P_{(i, j)}=2^{n+1}-1$; else " $P_{(i, j)}=5 \times 2^{n-1}$; end.

Case 4: $\left(3 \times 2^{n} \leq P_{(i, j)}<7 \times 2^{n-1}\right)$, then if $\left(3 \times 2^{n} \leq P_{(i, j)}<13 \times 2^{n-2}\right)$, then " $P_{(i, j)}=3 \times 2^{n}-1$; else " $P_{(i, j)}=7 \times 2^{n-1}$; end.

Step3: In this step when the $\mathrm{MSB}_{6}={ }^{\prime} 1$ ' and $\mathrm{EMB}={ }^{\prime} 0$ ', then the value pixels of cover image $P_{(i, j)}$ can be further segmented into four intervals as Eq. $(16,17)$, such that:

Interval $_{5}: 2^{n-1} \leq P_{(i, j)}<2^{n}$. Interval $6: 3 \times 2^{n-1} \leq P_{(i, j)}<2^{n+1}$

Interval $7: 5 \times 2^{n-1} \leq P_{(i, j)}<3 \times 2^{n}$. Interval ${ }_{8}: 7 \times 2^{n-1} \leq P_{(i, j)}<2^{n+2}$

From intervals $(5-8)$, the proposed APAP-MPOEE scheme required to divide each interval to the half $(1 / 2)$ derived as:

Case 5: $\left(2^{n-1} \leq P_{(i, j)}<2^{n}\right)$, then if $\left(2^{n-1} \leq P_{(i, j)}<3 \times 2^{n-2}\right)$, then " $P_{(i, j)}=2^{n-1}-1$; else if $(6 \leq n \leq 7)$, then " $P_{(i, j)}=2^{n}$; else " $P_{(i, j)}=2^{n-1}-1$; end; end.

Case 6: $\left(3 \times 2^{n-1} \leq P_{(i, j)}<2^{n+1}\right)$, then if $\left(3 \times 2^{n-1} \leq P_{(i, j)}<7 \times 2^{n-2}\right)$, then " $P_{(i, j)}=3 \times 2^{n-1}-1$; else if $(n==6)$, then " $P_{(i, j)}=2^{n+1}$; else " $P_{(i, j)}=3 \times 2^{n-1}-1$; end; end.

Case 7: $\left(5 \times 2^{n-1} \leq P_{(i, j)}<3 \times 2^{n}\right)$, then if $\left(5 \times 2^{n-1} \leq P_{(i, j)}<11 \times 2^{n-2}\right)$, then $P_{(i, j)}=5 \times 2^{n-1}-1$; 
else " $P_{(i, j)}=3 \times 2^{n}$; end.

Case 8: $\left(7 \times 2^{n-1} \leq P_{(i, j)}<2^{n+2}\right)$, then

if $\left(7 \times 2^{n-1} \leq P_{(i, j)}<15 \times 2^{n-2}\right)$, then " $P_{(i, j)}=7 \times 2^{n-1}-1$;

else " $P_{(i, j)}=7 \times 2^{n-1}-1$; end.

Step4: embedding algorithm of APAP-MPOEE $\mathrm{MSBn}_{\mathrm{n}}$ : Have been permuting the pixel of watermark image before inserted to protect the watermark bit and then set of the $\mathrm{MSB}_{6}$ of the cover image. Let's have a binary watermark $\mathrm{WL}_{(T, U)}$, then extracted the bits $\mathrm{EMB}=\left\{\mathrm{EMB}_{1}, \mathrm{EMB}_{2}, \ldots, \mathrm{EMB}_{(T \times U)}\right\}=$ $\operatorname{EMB}_{(i, j)}, n=6$ :

For $i=1$ to $M$

For $j=1$ to $N$

$$
\begin{aligned}
& \text { if }\left(\mathrm{MSB}_{6}==0 \& \mathrm{EMB}==0\right) \mid\left(\mathrm{MSB}_{6}==1 \& \mathrm{EMB}==1\right) \text {, then } \\
& \text { " } P_{(i, j)}=P_{(i, j)} ; \text { No change. } \\
& \text { else if }\left(\mathrm{MSB}_{6}==0 \text { and } \mathrm{EMB}==1\right) \text {, then } \\
& \text { if }\left(P_{(i, j)}>=0 \text { and } P_{(i, j)}<2^{\wedge} n-1\right) \text {, then ' } P_{(i, j)}=2^{\wedge} n-1 \text {; } \\
& \text { else if }\left(P_{(i, j)}>=2^{\wedge} n \text { and } P_{(i, j)}<3 \times 2^{\wedge} n-1\right) \text {, then } \\
& \text { if }\left(P_{(i, j)}>=2^{\wedge} n \text { and } P_{(i, j)}<5 \times 2^{\wedge} n-2\right) \text {, then } \\
& \text { " } P_{(i, j)}=\left(2^{\wedge} n\right)-1 \text {; } \\
& \text { else " } P_{(i, j)}=3 \times 2^{\wedge} n-1 \text {; end; } \\
& \text { else if }\left(P_{(i, j)}>=2^{\wedge} n+1 \text { and } P_{(i, j)}<5 \times 2^{\wedge} n-1\right) \text {, then } \\
& \operatorname{if}\left(P_{(i, j)}>=2^{\wedge} n+1 \text { and } P_{(i, j)}<9 \times 2^{\wedge} n-2\right) \text {, then } \\
& \text { " } P_{(i, j)}=\left(2^{\wedge} n+1\right)-1 \text {; } \\
& \text { else " } P_{(i, j)}=5 \times 2^{\wedge} n-1 \text {; end; } \\
& \text { else if }\left(P_{(i, j)}>=3 \times 2^{\wedge} n \text { and } P_{(i, j)}<7 \times 2^{\wedge} n-1\right) \text {, then } \\
& \operatorname{if}\left(P_{(i, j)}>=3^{\wedge} n \text { and } P_{(i, j)}<13 \times 2^{\wedge} n-2\right) \text {, then } \\
& \text { " } P_{(i, j)}=\left(3 \times 2^{\wedge} n\right)-1 \text {; } \\
& \text { else " } P_{(i, j)}=7 \times 2^{\wedge} n-1 \text {; } \\
& \text { end; end; end; end; end; }
\end{aligned}
$$

From the above algorithm we will applied under the algorithm FOBCB of the cover image with the random pixel manipulation. The proposed APAP-MPOEE-FOBCB ${ }_{\mathrm{MSB} 6}$ technique using as a embeds watermark bits in a boundary in corners board of the cover image and before embedding requires a checking between the $\mathrm{MSB}_{6}$ in the boundary in corners board pixel of the cover image and EMB of the embedded watermark bit, depending on the nearest of the adaptively pixel in the medial pyramid of embedding error to inform the watermarked image " $P_{(i, j)}$ obtained by a APAP-MPOEE-FOBCB ${ }_{\text {MSB6 }}$ scheme.

Step5: to extracted watermark bits from drawbacks in FOBCB of the watermarked image by using inverse the same procedure of the embedded algorithm without using the steps of embedding process in proposed method adjust recovery the watermark bits from the $\mathrm{FOBCB}$ in watermarked image depending on the sequence number to know the manipulation pixel between boundary corners board in the watermarked image and then select one of drawbacks in the $\mathrm{MSB}_{6}$, after extracted watermark required to rearranging the change of the pixel, then the watermark in original form is thus obtained.

Theoretical analysis of the proposed method we know that by applying the embedding algorithm of the $\mathrm{FOBCB}_{\mathrm{MSB} 6}$, the embedding error $\Omega^{\prime}=\left|P_{(i, j)}-P_{(i, j)}\right|$ by directly replacement of embed watermark bit $=2^{\mathrm{n}-1}=32$. From the analysis of previous works and modified algorithms. Thus when applying proposed method the embedded error $\Omega=\mid$ " $P_{(i, j)} P_{(i, j)} \mid$ in the case. 1 within interval $2^{n-2} \leq P_{(i, j)}<2^{n-1}$, where are the $\Omega$ in range from $1 \leq \Omega \leq 16$. It has the same $\Omega$ in case. $(2,3,4,5,6$ and 7$)$ the embedding errors $\Omega$ increased one by one in the range from $2^{n-2} \leq P_{(i, j)}<15^{\times} 2^{n-2}$, where is each case minimizing the embedding errors to the medial pyramid of embedding error to inform the watermarked pixel, by the way shown in the sketched of the Fig. 1, each case sketched the pyramid of embedding error are minimized to the half when the values of gray scale in cover image pixel $P_{(i, j)}$ in the interval between $2^{n-2} \leq P_{(i, j)} \leq 15^{\times} 2^{n-2}$. as shown in Fig. 1 , where are the $\Omega$ are minimized to the half $\left(2^{n-2}\right)$ of the Max-embedding error $=\left(2^{n-1}\right)$ with compared by previous works and modified algorithms was found better. Otherwise the $\Omega$ are growth one by one according to the values of gray scale in cover image pixel $P_{(i, j)}$ in the range from $17 \leq \Omega \leq 32$ as shown in the first half of case. 1 and in the last half in case.8, when the values of gray scale in cover image pixel $P_{(i, j)}$ in the intervals from $0 \leq P_{(i, j)}<2^{n-2}$ within case. 1 and from $15 \times 2^{n-2}<P_{(i, j)}<256$ within case.8. Theoretically can be calculated the summation of embedding errors ' $i$ ' in all intervals, but will be neglects the gray scale values $P_{(i, j)}$ from intervals $0 \leq P_{(i, j)}<2^{n-2}$ and from $15 \times 2^{n-2}$ $<P_{(i, j)}<256$, where almost of gray scale images are out of these intervals, can be derived ' $i$ ' as:

$$
\sum_{i=1}^{2^{n-2}} i=1+2+\ldots+2^{n-2}=\frac{2^{n-2}\left(2^{n-2}+1\right)}{2}=2^{n-3}\left(2^{n-2}+1\right)
$$

From Eq.(18) the can be calculated the average of embedding errors derived by:

$$
\text { The average of embedding error }=\frac{2^{n-3}\left(2^{n-2}+1\right)}{2^{n-2}}=\frac{2^{n-2}+1}{2}
$$

Theoretically, the averg.WMSE between the cover and watermarked image can be derived by Eq.(5):

$$
\text { Averg. } \operatorname{WMSE}^{*}=\frac{1}{M \times N} \sum_{i=1}^{M} \sum_{j=1}^{N}\left[\frac{2^{n-2}+1}{2}\right]^{2}=\left[\frac{2^{n-2}+1}{2}\right]^{2}
$$

The WMSE $=\left(2^{n-1}\right)^{2}$ by the direct replacement by the simple $\mathrm{LSB}_{n}$ and $\mathrm{MSB}_{n}$ substitution method are constant of embedded error $=2^{n-1}$. But with proposed method the $\operatorname{Max} \cdot \operatorname{WMSE}^{*}=\left(2^{n-2}\right)^{2}, \quad \operatorname{Min} \cdot \mathrm{WMSE}^{*}=1, \quad$ and the average.WMSE are obtained in Eq.(20). Theoretically, by combining WMSE and Max.WMSE*, we have

$$
\frac{\text { Max.WMSE }}{\text { WMSE }}=\frac{\left(2^{n-2}\right)^{2}}{\left(2^{n-1}\right)^{2}} \quad, \quad \text { Max.WMSE } E^{*}=\frac{\left(2^{n-2}\right)^{2}}{\left(2^{n-1}\right)^{2}} W M S E
$$


From Eq.(21) and when $\mathrm{n}=6$ reveals that the $M a x \cdot W M S E^{*}=\frac{1}{4} W M S E$ this result of our analysis shows that the average of embedding errors in Eq. $(20)=8.5_{\text {avrg }}$ and $\mathrm{WMSE}^{*}$ are proved efficient and better than obtained by the previous works and modified algorithms.

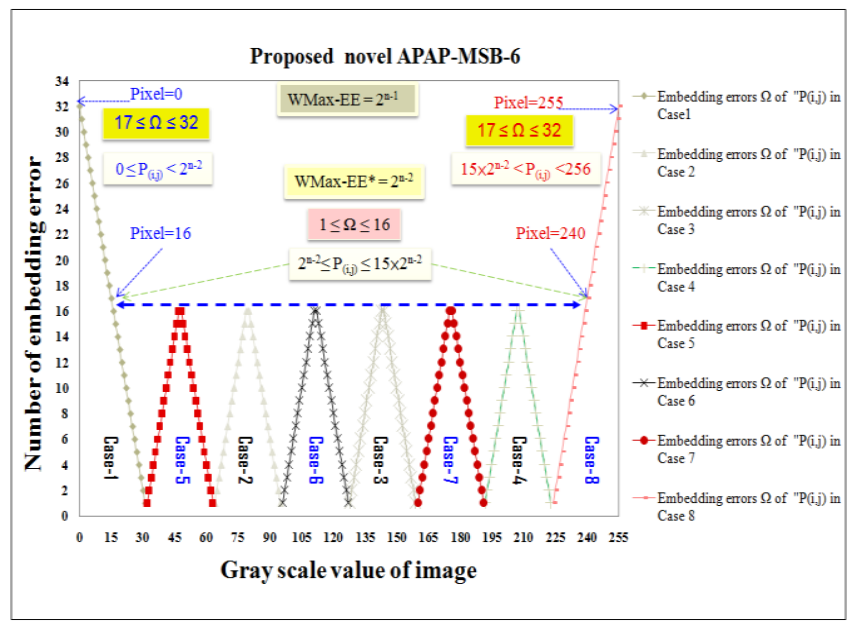

Fig. 1. Proposed watermarking technique " $P_{(i, j)}$ compared with the ' $P_{(i, j)}$.

\section{PERFORMANCE RESUlts WITH Discussion}

The experimental results have been measured by comparative study between the previous works, proposed modified algorithms and proposed method (i)-Theoretical analysis. (ii)-Applied on the different benchmark.

\section{A. Theoretical Analysis}

Theoretically, suppose that all the pixels in the cover image are used for the embedding of watermark bit, then have been measure the Max, Min and average number of embedding errors $\Omega, W M S E, W M S E^{*}$ and $P S N R_{\text {worst }}$ between the cover and watermarked image, the Table. (I-II) tabulates the comparisons results, the worst number of embedding errors $\Omega$ and PSNR worst, It could be seen that the image quality of the watermarked image is degraded drastically when $n$ growth up one by one In this letter, the number of embedding errors $\Omega$ in proposed method set-of-MSB $6=16 \mathrm{Max}, 8.5_{\mathrm{avrg}}$, $1 \mathrm{Min}$ and the WMSE* in MSB6 $=256$ Max, 72.25 avrg and 1 Min are proved and it was lowest than with compared of the previous works and modified algorithms, the Table. II tabulates the PSNR worst in proposed method set of $\mathrm{MSB}_{6}=(24.048(\mathrm{~dB}))$ Max, $(29.542(\mathrm{~dB}))_{\text {avg }}$, and $(48.130(\mathrm{~dB}))_{\text {Min }}$ are higher are proved and was found better. Finally, from theoretically analysis the proposed method are proved efficient and better than obtained by the previous works and modified algorithms.

\section{B. The Experimental Result Applied on Different Benchmarks}

The experimental results have been applied on different benchmark six-test-images (Lena, Boat, Baboon, jet, Birds and Pills) and two quantum of watermark bit embedded with different size of $45 \times 45$ and $16 \times 16$ to study the performance of enhancement grey scale image quality (fidelity), imperceptibility, capacity and robustness under different attacks. In order to compare the performance results of the proposed method APAP-MPOEE-FOBCB ${ }_{\mathrm{MSB} 6}$, with the state-of-the-art-algorithms are required[9], [11], [13], [14], [15] and with modified algorithms by applying the FOBCB $_{\mathrm{MSB} 6}[15]$. A set of standard six-test grey scale images $_{(512 \times 512)}$ has been used as a cover images as shown in Table.III. However the Max-bits can be embedded 2048bits in the cover image.

TABLE I: THE NUMBER OF EMBEDDING ERRORS AND COMPARISON

\begin{tabular}{|c|c|c|c|c|c|c|c|c|c|c|}
\hline \multirow{2}{*}{$\begin{array}{l}\text { Comparison between } \\
\text { Methods of embedded } \\
\text { watermark bits: }\end{array}$} & \multirow[b]{2}{*}{ Level } & \multirow{2}{*}{$\begin{array}{l}\text { The number of embedding } \\
\text { errors, } 1<\mathbf{n} \leq \$, P_{(i j)} \neq p^{\prime}(i \mathcal{i})\end{array}$} & \multicolumn{8}{|c|}{$\begin{array}{l}\text { There are only } 256 \text { possible pixel values for eight-bit gray } \\
\text { scale images, Theooretically, the number of embedding errors }\end{array}$} \\
\hline & & & $\bar{g}$ & 5 & $\bar{E}$ & $\overline{\underline{y}}$ & $\frac{3}{y}$ & $\frac{3}{p}$ & $\frac{3}{y}$ & $\frac{3}{2}$ \\
\hline $\begin{array}{l}\text { Simple L } \\
\text { substitu }\end{array}$ & Constant & $2^{n-1}$ & 1 & 2 & 4 & 8 & 16 & 32 & 64 & 128 \\
\hline \multirow{3}{*}{$\begin{array}{l}\text { Method of Wang-Lin-Lin } \\
\text { scheme } \\
\& \\
\text { PAP-algorithm-1 set of } \\
\text { MSB }_{6}\end{array}$} & Max & $2^{n-1}$ & 1 & 2 & 4 & 8 & 16 & 32 & 64 & 128 \\
\hline & Average & $\frac{2\left(2^{n-1}-2^{n-3}+1\right)-1}{2}$ & 1 & 2 & 3.5 & 6.5 & 12.5 & 24.5 & 48.5 & 96.5 \\
\hline & Min & $2^{\mathrm{n}-2}+1$ & 1 & 2 & 3 & 5 & 9 & 17 & 33 & 65 \\
\hline $\begin{array}{l}\text { Simple k-LSB substitution } \\
\text { method using 'k' capacity of } \\
\text { embedded watermark bits }\end{array}$ & Constant & $2^{\mathrm{k}}-1$ & 1 & 3 & 7 & 15 & 31 & 63 & 127 & 255 \\
\hline \multirow{3}{*}{$\begin{array}{l}\text { Method of Chi-Kwong- } \\
\text { L.M.Cheng scheme, using ' } \mathrm{k} \text { ' } \\
\text { capacity of embedded } \\
\text { watermark bits in k-LSB }\end{array}$} & Max & $2^{\mathrm{k}-1}$ & 1 & 2 & 4 & 8 & 16 & 32 & 64 & 128 \\
\hline & Average & $\frac{2^{k-1}+1}{2}$ & 1 & 1.5 & 2.5 & 4.5 & 8.5 & 16.5 & 32.5 & 64.5 \\
\hline & Min & 1 & 1 & 1 & 1 & 1 & 1 & 1 & 1 & 1 \\
\hline $\begin{array}{c}\text { PAP-algorithm-2 set of } \\
\text { MSBB }_{6}\end{array}$ & Constant & $2^{n-1}$ & 1 & 2 & 4 & 8 & 16 & 32 & 64 & 128 \\
\hline \multirow{3}{*}{$\begin{array}{l}\text { Method of Aiad and Abdul } \\
\text { scheme } \\
\& \\
\text { PAP-algorithm-3 set of } \\
\text { NSB }_{6}\end{array}$} & Max & $2^{n-1}$ & 1 & 2 & 4 & 8 & 16 & 32 & 64 & 128 \\
\hline & Average & $\frac{2^{n-1}+1}{2}$ & 1 & 1.5 & 2.5 & 4.5 & 8.5 & 16.5 & 32.5 & 64.5 \\
\hline & Min & 1 & 1 & 1 & 1 & 1 & 1 & 1 & 1 & 1 \\
\hline \multirow{3}{*}{ Proposed method } & Max & $2^{\mathrm{n}-2}$ & 1 & 1 & 2 & 4 & 8 & 16 & 32 & 64 \\
\hline & Average & $\frac{2^{n-2}+1}{2}$ & 1 & 1 & 1.5 & 2.5 & 4.5 & 8.5 & 16.5 & 32.5 \\
\hline & Min & 1 & 1 & 1 & 1 & 1 & 1 & 1 & 1 & 1 \\
\hline
\end{tabular}

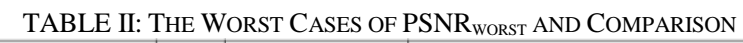

\begin{tabular}{|c|c|c|c|c|c|c|c|c|c|c|}
\hline \multirow{2}{*}{$\begin{array}{l}\text { Comparison between } \\
\text { Methods of embedded } \\
\text { watermark bits: }\end{array}$} & \multirow[t]{2}{*}{ Level } & \multirow{2}{*}{ 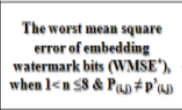 } & \multicolumn{8}{|c|}{\begin{tabular}{|l} 
Suppose that all the pirels in the cover image are used for the \\
embedded watermark bits by the lis of substitution \\
methods. Theoretically, in the wornt PSNR (AB) are:
\end{tabular}} \\
\hline & & & 5 & 5 & 5 & 5 & $\frac{3}{x}$ & $\frac{z}{\not z}$ & 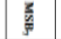 & $\frac{x}{\partial y}$ \\
\hline & nstant & $\left(2^{n-1}\right)^{2}$ & 48.130 & 42.110 & 36.099 & 30.069 & 24.048 & 18.027 & 12.007 & 5.986 \\
\hline \multirow{3}{*}{$\begin{array}{c}\text { Method of Wang-Lin-Lin } \\
\text { scheme } \\
\& \\
\text { PAP-algorithm-1 set of } \\
\text { MSB }_{6}\end{array}$} & ax & $\left(2^{n-1}\right)^{4}$ & 130 & 42.110 & 36.099 & 30.069 & 24.048 & 18.027 & 12.007 & 5.9866 \\
\hline & Average & {$\left[\frac{2\left(2^{*-1}-2^{*-1}+1\right)-1}{2}\right]$} & 48.130 & 42.11 & 9 & 31.872 & 26.192 & 20.347 & 14.415 & 8.4402 \\
\hline & Min & $\left(2^{n-2}+1\right)^{2}$ & 48.130 & 42.110 & 38.588 & 34.151 & 29.045 & 23.521 & 17.760 & 11.872 \\
\hline $\begin{array}{l}\text { Simple k.LSB substitution } \\
\text { method using 'k' capacity of } \\
\text { embedded watermark bits }\end{array}$ & ustant & $\left(2^{k}-1\right)^{2}$ & 8.130 & 38.588 & 31.228 & 24.608 & 18.303 & 12.143 & 6.054 & 0 \\
\hline \multirow{3}{*}{$\begin{array}{l}\text { Method of Chi-Kwong- } \\
\text { L.M.Cheng scheme, using 'k' } \\
\text { capacity of embedded } \\
\text { watermark bits in ke.LSB }\end{array}$} & & $\left(2^{k-1}\right)^{2}$ & 130 & 42.110 & 9 & 30.069 & 24.048 & 18.027 & 12.0072 & 59866 \\
\hline & Average & $\left.\frac{2^{k-1}+1}{2}\right]$ & 48.130 & 44.608 & 40.1 & 35.066 & 29.542 & 23.781 & 17.893 & 11.939 \\
\hline & Min & 1 & 48.130 & 48.130 & 48.130 & 48.130 & 48.130 & 48.130 & 48.130 & 48.130 \\
\hline $\begin{array}{l}\text { PAP-algorithm-2 set of } \\
\text { MSB }_{6}\end{array}$ & Contant & $\left(2^{n+1}\right)^{7}$ & 48.130 & 42.1 & 36.099 & 30.069 & 24.048 & 18.027 & 12.00 & 295 \\
\hline \multirow{3}{*}{$\begin{array}{c}\text { Method of Aiad and Abdul } \\
\text { scheme } \\
\& \\
\text { PAP-algorithm-3 set of } \\
\text { MSB }_{6}\end{array}$} & Ma: & $\left(2^{n-1}\right)^{2}$ & 130 & 110 & 9 & 30.069 & 24.048 & 18.027 & 2.0072 & 5 \\
\hline & Average & $\left.\frac{2 n-1+1}{2}\right]$ & 48 & 4.608 & 40.172 & 35.066 & 29.542 & 23.781 & 17.893 & 11.939 \\
\hline & Min & 1 & 48.130 & 48.130 & 48.130 & 48.130 & 48.130 & 48.130 & 48.130 & 48.130 \\
\hline \multirow{3}{*}{ Proposed method } & Max & $\left(2^{n-2}\right)^{2}$ & 48.130 & 48.130 & 42.110 & 36.099 & 30.069 & 24.048 & 18.027 & 12.007 \\
\hline & Average & $\frac{2 n-2+1}{2}$ & 48.130 & S150 & 608 & 80.172 & 35.066 & 29.542 & 23.7811 & 17.8981 \\
\hline & Min & 1 & 48.130 & 48.130 & 48.130 & 48.130 & 48.130 & \begin{tabular}{|l|l|}
48.130 \\
\end{tabular} & 48.130 & 48.130 \\
\hline
\end{tabular}

\section{1) Imperceptibility}

The PSNR is typically used and it is a standard way to measure image fidelity, derived by:

$$
P S N R=10 \log _{10} \frac{255^{2}}{M S E} \quad d B
$$

The Table. III tabulates the performance results of PSNR, in the proposed watermarking technique is getting a higher of PSNR equal $\left(52.6403_{\mathrm{dB}}\right)_{\mathrm{avrg}}$, with compared of all the list substitutions techniques, it was found better, this is the prove imperceptibility and image fidelity.

\section{2) Robustness with discussion}

We evaluated robustness of the proposed method under major attacks of digital signal processing operations: 
watermark degrading attacks [16], removal attacks [12], and geometric transformations attacks[7], [10], [12], [17]. We measured the similarity between the original watermark $W_{(i, j)}$ and the watermark extracted $W_{(i, j)}$ from the attacked image by: normalized cross correlation (NCC) and similarity function (SM)[12], [17], where the similarity values of NCC and SM of about 0.75 or above is considered acceptable[3], [16], [18], can be derived by:

$$
N C C=\frac{\sum_{i=1}^{M} \sum_{j=1}^{N}\left(W_{(i, j)} \times W_{(i, j)}^{\prime}\right)}{\sum_{i=1}^{M} \sum_{j=1}^{N} W_{(i, j)}{ }^{2}} \quad, \quad S M=\frac{\sum_{i=1}^{M} \sum_{j=1}^{N}\left(W_{(i, j)} \times W_{(i, j)}^{\prime}\right)}{\sqrt{\sum_{i=1}^{M} \sum_{j=1}^{N} W_{(i, j)}{ }^{2} \times \sum_{i=1}^{M} \sum_{j=1}^{N} W_{(i, j)}^{\prime}}}
$$

TABLE III: THE PERFORMANCE RESULTS OF PSNR

\begin{tabular}{|c|c|c|c|c|c|c|c|}
\hline \multirow{2}{*}{$\begin{array}{c}\text { Comparisouns between the proposed } \\
\text { watermarking technique and modified } \\
\text { algovithms within the previous works in the } \\
\text { literature: }\end{array}$} & \multicolumn{7}{|c|}{ Peak signal to noise ratio (dB) measured on different benchmark of test images } \\
\hline & Lena & Boat & Baboon & jet & Birds & $\begin{array}{l}\text { Page } \\
\text { Pills } \\
\text { Pill }\end{array}$ & Average \\
\hline The optimal moderately $\mathrm{LSB}_{4}$ scheme $[12]$ & 38.925852 & 39.098428 & 39.051341 & 39.012303 & 39.057997 & 39.079512 & 39.04642938 \\
\hline The OPA.AP K.LSB 1 scheme, $\mathrm{k}=1 \quad[3]$. & 51.402418 & 51.51654 & 51.524969 & 51.523413 & 51.555809 & 51.536459 & 51.33140408 \\
\hline The OP.AP K.SSB 1,2 scheme, $\mathrm{k}=2 \quad[\mathrm{~s}]$. & 46.703865 & 46.698703 & 46.727954 & 46.708782 & 46.715988 & 46.739086 & 46.71989642 \\
\hline The OP.AP $\mathrm{K}-\mathrm{LSB}_{1,23}$ scheme, $\mathrm{k}=3$ [3]. & 40.690663 & 40.498567 & 40.544923 & 40.498947 & 40.494408 & 40.589771 & 40.53400146 \\
\hline The OP.AP K.LSB $]_{1,3,4}$ scheme $\mathrm{k}=4$ [3]. & 34.527257 & 34.603451 & 34.453300 & 34.407339 & 34.449637 & 34.480819 & 34.45619054 \\
\hline $\begin{array}{l}\text { Hiding data in the } \mathrm{LSB}_{3} \text { substitution with } \\
\text { modified } \mathrm{LPAP} \text {, the LS } \mathrm{B}_{1,2} \text { scheme [4]. }\end{array}$ & 42.290906 & 42.401492 & 42.355747 & 42.307101 & 42.417782 & 42.366067 & 42.36186646 \\
\hline $\begin{array}{l}\text { The scheme of an investigation watermarking } \\
\text { into the simple MSBB-6 substitution [5]. }\end{array}$ & 21.031317 & 20.997112 & 21.087254 & 20.894387 & 21.034428 & 20.974197 & 20.97653196 \\
\hline $\begin{array}{l}\text { The watermarking techniquebased in the } \\
\text { FOBCB set of the MSB } \text { S }_{6} \text { scheme }[7] \text {. }\end{array}$ & 42.292088 & 42.221898 & 42.038696 & 42.195866 & 42.217548 & 42.105965 & 42.17451396 \\
\hline $\begin{array}{l}\text { Proposed modified(PAP-algorithm-1) } \\
\text { Applying algovithm of the optimal moderately } \\
\text { in the FOBCB set of the MSB } \text { B }_{6} \text { scheme. }\end{array}$ & 43.014402 & 42.984100 & 43.378092 & 44.267315 & 44.584894 & 43.951791 & 44.12519142 \\
\hline $\begin{array}{l}\text { Proposed modified(PAP-algorithm-2) } \\
\text { Applyingalgorithm of the OPAPin the } \\
\text { FOBCB set of the MSB } \text { S }_{6} \text { scheme. }\end{array}$ & 42.292088 & 42.221898 & 42.038696 & 42.195866 & 42.217548 & 42.105965 & 42.17451396 \\
\hline 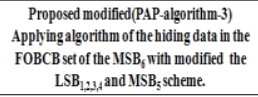 & 46.789861 & 46.133148 & 46.259490 & 46.580212 & 45.623759 & 46.053467 & 46.12436588 \\
\hline $\begin{array}{c}\text { Proposed watermarkingtecthnique } \\
\text { of the APAP-FOBCB set of the } M \mathrm{SS}_{6} \text { scheme. }\end{array}$ & 53.299495 & 51.897225 & 52.485536 & 53.026103 & 52.388548 & 52.540476 & 52.64033938 \\
\hline
\end{tabular}

1) The watermark degrading attacks: for adding the Gaussian noise, Salt \& Pepper noise and Speckle noise to the watermarked image, can be used as an attacks to remove the watermark. Effect Gaussian noise: In this experiment results as shown in Table. IV. The observations of the proposed method are robust under effect of Gaussian noise attacks by applying proposed method can be extracted watermark with acceptable degree: $\mathrm{NCC}=(0.895)_{\mathrm{avrg}}$, and $\mathrm{SM}=(0.907)_{\mathrm{avrg}}$, with the extracted watermark $16 \times 16$, and $\mathrm{NCC}=(0.840)_{\mathrm{avrg}}$, and $\mathrm{SM}=(0.857)_{\mathrm{avrg}}$, with watermark ${ }_{45 \times 45}$. Effect Salt \& Pepper noise attacks: In this experiment results as shown in Table. V. The observations of the proposed method are robust under effect of Salt \& Pepper noise attacks by applying proposed method can be extracted watermark with acceptable degree: $\mathrm{NCC}=(0.988)_{\mathrm{avrg}}$, and $\mathrm{SM}=(0.991)_{\mathrm{avrg}}$, with the extracted watermark ${ }_{16 \times 16}$, and $\mathrm{NCC}=(0.971)_{\mathrm{avrg}}, \quad$ and $\mathrm{SM}=(0.974)_{\mathrm{avrg}}$, with watermark ${ }_{45 \times 45}$. Effect speckle noise attacks: In this experiment results as shown in Table. V. The observations of the proposed method are robust under effect of Salt \& Pepper noise attacks by applying proposed method can be extracted watermark with acceptable degree: $\mathrm{NCC}=(0.904)_{\mathrm{avrg}}$, and $\mathrm{SM}=(0.917)_{\mathrm{avrg}}$, with the extracted watermark ${ }_{16 \times 16}$, and $\mathrm{NCC}=(0.843)_{\mathrm{avrg}}$, and $\mathrm{SM}=(0.861)_{\mathrm{avrg}}$, with watermark $45 \times 45$.

2) Geometric transformations attack: the performance results are shown in the Table. $\mathrm{V}$ can be extracted watermark with acceptable degree under: Re-scaling: is tested by first resizing the watermarked to the scaled factor $60 \%$ of its size and then enlarging the image to its original size, it are obtained $\mathrm{NCC}=(0.883)_{\mathrm{avrg}}$, and $\mathrm{SM}=(0.891)_{\mathrm{avrg}}$, with the extracted watermark ${ }_{16 \times 16}$, and $\mathrm{NCC}=(0.846)_{\mathrm{avrg}}, \quad$ and $\mathrm{SM}=(0.837)_{\mathrm{avrg}}, \quad$ with watermark ${ }_{45 \times 45}$. Re-rotation: is tested by first rotate the watermarked small angle rotation $30^{\circ} \mathrm{CW}$ and then re-rotate the watermarked to the same angle rotation $30^{\circ} \mathrm{CCW}$ to its original size, it are obtained $\mathrm{NCC}=(0.899)_{\mathrm{avrg}}$, and $\mathrm{SM}=(0.879)_{\mathrm{avrg}}$, with the extracted watermark $_{16 \times 16}, \quad$ and $\mathrm{NCC}=(0.812)_{\mathrm{avrg}}, \quad$ and $\mathrm{SM}=(0.755)_{\mathrm{avrg}}$, with watermark ${ }_{45 \times 45}$. JPEG: is currently one of the most widely used. The results are obtained after compressed $\mathrm{NCC}=(0.750016)_{\mathrm{avrg}}, \quad$ and $\mathrm{SM}=(0.750153)_{\mathrm{avrg}}$, with the extracted watermark ${ }_{16 \times 16}$, and $\mathrm{NCC}=(0.55674)_{\mathrm{avrg}}$, and $\mathrm{SM}=(0.58218)_{\mathrm{avrg}}$, low robust with watermark $\mathrm{k}_{45 \times 45}$.

3) Changing in lower order bit of gray values called the watermark removal attack, the attacker would only have to replace all LSB bits with a '1' fully defeating the effects or complement the LSB bits and the watermark cannot be recovered from lower order bits $\operatorname{LSB}_{1,2,3,4}$, the proposed method prevents the attacker to remove watermark bit.

TABLE IV: THE RESULTS OF DEGRADING ATTACKS

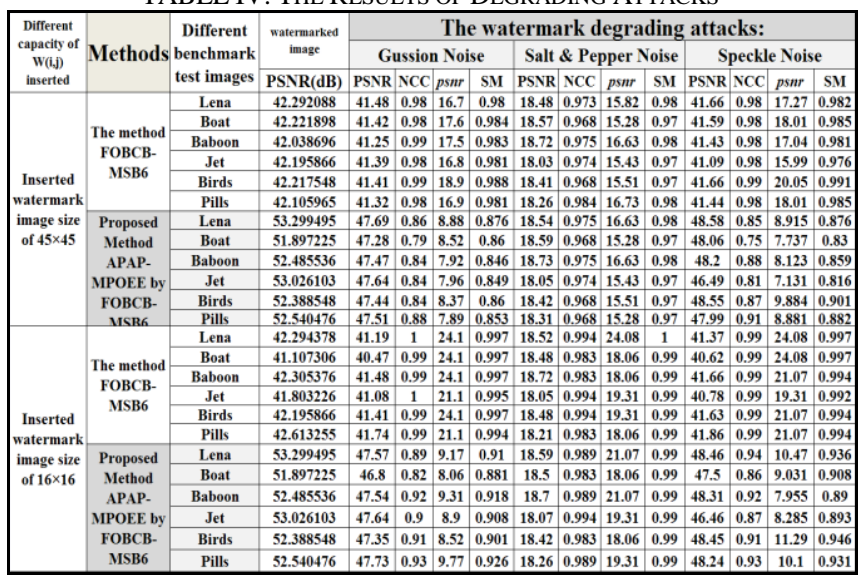

TABLE V: THE RESULTS OF GEOMETRIC TRANSFORMATIONS ATTACKS

\begin{tabular}{|c|c|c|c|c|c|c|c|c|c|c|c|c|}
\hline \multirow{3}{*}{$\begin{array}{c}\text { Different } \\
\text { capacity of } \\
\text { W(i,j) } \\
\text { inserted }\end{array}$} & \multirow{3}{*}{ ethods } & \multirow{3}{*}{$\begin{array}{c}\text { Different } \\
\text { benchmark } \\
\text { test images }\end{array}$} & \multicolumn{10}{|c|}{ Geometric transformations attacks } \\
\hline & & & \multicolumn{3}{|c|}{ Scaling $(60 \%)$} & \multicolumn{3}{|c|}{ Rotation $30^{\circ} \mathrm{CW}$} & \multicolumn{4}{|c|}{ Geometric distortion } \\
\hline & & & & psur & & NCC & $s n r$ & SM & & & $p s n r$ & $\mathrm{~s}$ \\
\hline \multirow{10}{*}{$\begin{array}{c}\begin{array}{c}\text { Inserted } \\
\text { watermark } \\
\text { image size of } \\
45 \times 45\end{array} \\
\end{array}$} & \multirow{5}{*}{$\begin{array}{l}\text { The method } \\
\text { FOBCB- } \\
\text { MSB6 }\end{array}$} & Lena & 0.846 & 7.59 & 0.84 & 0.809 & 5.44 & 0.75 & 37.34 & 0.8 & 6.64 & 08 \\
\hline & & & & 750 & & & & 075 & & & & 87 \\
\hline & & Baboc & 846 & 7.59 & 84 & 816 & 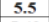 & .76 & & 81 & 791 & \\
\hline & & & & 7.59 & 84 & & $\frac{2}{4}$ & $\frac{75}{77}$ & & \begin{tabular}{|l|l|l}
0.86 \\
0.83
\end{tabular} & 098 & \\
\hline & & & & & & & & & & & & \\
\hline & \multirow{6}{*}{$\begin{array}{l}\text { Proposed } \\
\text { Method } \\
\text { APAP- } \\
\text { MPOEE by } \\
\text { FOBCB- } \\
\text { MSB6 }\end{array}$} & & & & & & & & & & & \\
\hline & & & & 7.59 & & & 57 & & & 0.4 & & \\
\hline & & & & & & & & & & & & \\
\hline & & & & & & & 42 & 75 & & & 61 & \\
\hline & & & & & & & & & & & & \\
\hline \multirow{11}{*}{ 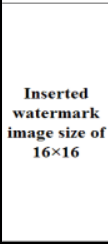 } & & & & & & & & & & 39 & & 8 \\
\hline & \multirow{5}{*}{$\begin{array}{l}\text { The method } \\
\text { FOBCB- } \\
\text { MSB6 }\end{array}$} & & & & & & 27 & 87 & & 9 & 311 & \\
\hline & & & 0.884 & 8.17 & & & & & & & & \\
\hline & & & & & & & & & & & & \\
\hline & & & & & & & & & & & & \\
\hline & & & & & & & & & & & & \\
\hline & \multirow{5}{*}{$\begin{array}{c}\text { Proposed } \\
\text { Methed } \\
\text { APAP- } \\
\text { MPOEE by } \\
\text { FOBCB- } \\
\text { MSB6 } \\
\end{array}$} & & & & 39 & & 7.65 & 88 & & 75 & .54 & \\
\hline & & & & & & & 55 & 87 & 6 & 75 & 26 & \\
\hline & & & & & & & & & & & & 75 \\
\hline & & & 84 & 8. & 89 & & 65 & 89 & & 76 & 638 & 76 \\
\hline & & & .884 & 8.17 & 0.89 & 112 & 7.8. & 0.8 & 43.86 & \begin{tabular}{|l|l|} 
& 0.82 \\
\end{tabular} & 4.58 & 0.7 \\
\hline
\end{tabular}

\section{CONCLUSION}

The digital watermarking technology is a way to apply digital information hiding techniques to prevent attacks to detect hidden information, have been proposed a new novel 
fidelity and robust technique that satisfies the requirements and statement problem. Experimental results was computed by, First: Theoretical analysis are proved effectiveness and better than obtained by the previous works and modified algorithms in the average of worst case, minimized the embedding error to the medial pyramid of embedding error, getting a Max:WMSE $^{*}=\frac{1}{4} W M S E$ and higher in the worst case of PSNR $\left.=\left(24.048_{(\mathrm{dB})}\right)_{\text {Max }}\right\}$. Second: Appling on the different benchmark are compared with an previous works and modified algorithms was found better and good robustness with extracted watermark from degrading, removal and geometric transformations attacks to an acceptable degree Finally, from our study of previous works and modified the algorithms, the performance results are obtained the modified PAP-algorithm-3 great a good performance in $\mathrm{PSNR}=\left(46.23_{\mathrm{dB}}\right)_{\mathrm{avrg}}$ with compared of the previous works.

\section{REFERENCES}

[1] J. Cox, M. L. Miller, and J. A. Bloom, Digital Watermarking Morgan Kaufmann Series in Multimedia Information and Systems, Elsevier, San Francisco, Copyright. 2002 by Academic Press, United States of America, 2002.

[2] C.-S. Lu, "Multimedia security: Steganography and digital watermarking techniques for protection of intellectual property," Idea Group Inc (Pub: Hershey, London, Melbourne, Singapore), 2005.

[3] A.-H. Ali, "Combined DWT-DCT digital image watermarking," The Journal of Computer Science, vol. 3, no. 9, pp. 740-746, 2007.

[4] E. A. Bansall and S. S. Bhadouria, "Network security and confidentiality with digital watermarking," in proc. of 2007 Inaugural IEEE International Conference on Digital Ecosystems and Technologies, Cairns Australia, pp. 325-328, 2007.

[5] F. A. P. Petitcolas, "Watermarking schemes evaluation," IEEE Sig. Pro. Mag., vol. 17, no. 5, pp. 58-64, Sep. 2000.

[6] R. M. Lesley, G. M. Stephen, G. S. Talal, E. T. Robert, and Z. Francis, "Robustness and Security of digital watermarks," in Proc. of the Sec. Inter. Conf. on Financial Cryptography, Lecture Notes in Comp. Sc., Pub: Springer London, UK, vol. 1465, pp. 227-240, 1998.

[7] E. Cole, Hiding in Plain Sight: Steganography and the Art of Covert Communication, Published by Wiley Publishing, Inc., Indianapolis, Indiana, Copyright (C) 2003.

[8] S. Katzenbeisser and F. A. P. Petitcolas, Information Hiding Techniques for Steganography and Digital Watermarking, Copyright (C)2000 Artech house, inc.

[9] A. Ibraheem and A. Sada, "Hiding data using LSB-3," The J. Basrah Researches Sc., vol. 33. no.4, pp. 81-88, 19/DEC/ 2007.
[10] L. Liu, "A survey of digital watermarking technologies," Technical Report, Computer Vision Laboratory, Department of Electrical and Computer Engineering, State University of New York at Stony Brook, NY, USA, 2005.

[11] R.-Z. Wang, C.-F. Lin, and J.-C. Lin, "Hiding data in images by optimal moderately significant-bit replacement," IEEE Electronics Letters. vol. 36, no. 25, pp. 2069-2070, 28 Dec. 2000.

[12] S. P. Maity and M. K. Kundu, "Robust and blind spatial watermarking in digital image," in Proc. of the Third Indian Conference on Computer Vision, Graphics and Image Processing, Ahmadabad, India, pp. 388-393, Dec 16-18, 2002.

[13] C.-K. Chan and L. M. Cheng, "Hiding data in images by simple LSB substitution," The J. Pattern Recog. Socy., vol. 37, pp. 469-474, 2004.

[14] K. Curran, X. L. Li, and R. Clarke, "An investigation into the use of the least significant bit substitution technique in digital watermarking," American Journal of Applied Science, vol. 2, no. 3, pp. 648-654, 2005.

[15] M. B. Aliwa, T. E. A. E. Tobely, M. M. Fahmy, M. E. S. Nasr, and M H. A. E. Aziz, "Robust digital watermarking based falling-off-boundary in corners board-MSB-6 gray scale images," IJCSNS Inter. J. of Computer Sc. and Net. Sec, vol. 9, no. 8, pp. 227-240, 30 Aug 2009.

[16] M. S. Shaikh and Y. Dot, "A watermarking scheme for digital images using multilevel wavelet de compodition," Mal. J. of Comp. Sc., vol. 16, no. 1, pp. 24-36, Jun 2003.

[17] M. Kutter, F. A. P. Petitcolas, "Fair evaluation methods for image watermarking systems," Journal of Electronic Imaging, vol. 9, no. 4, pp. 445-455, Oct. 2000.

[18] K. Hameed, A. Mumtaz, and S. A. M. Gilani, "Digital image watermarking in the wavelet transform domain," in Proc. of World Academy of Science, Engineering and Technology, World Information Society, vol. 13. pp. 86-89, May 2006.

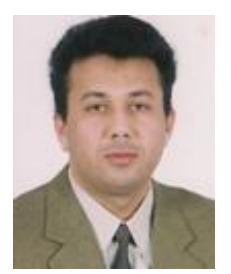

Mehemed Bashir Aliwa received the B.Sc. Degree in Computer Engineering on September 1, 1992 from the Engineering Academy, Tajoura-Libya and the M.Sc. Degree in Computer Engineering on March.2008 from the Arab Academy for Science, Technology and Maritime Transport College of Engineering and Technology, Alexandria-Egypt. $\mathrm{He}$ is currently pursuing the Ph.D. degree at the Electrical Engineering (Computer and Control Engineering) of the Faculty of Engineering TANTA University, Egypt. From 1992 to 1996 his was working in the research center of military industrialization, from 1996 to 1997 as a Lecturer at the School of Electronic Support and from 1997 to 2005 as a director, office of the global information network and the office of training and maintenance in computer system in Authority operations and training Libyan armed forces. His research interests include hiding information, digital watermarking and routing protocol in mobile ad-hoc networks. 